\title{
PARTON DYNAMICS AT LOW $X$ USING DIS 3-JET EVENTS
}

\author{
C. WERNER \\ Physikalisches Institut, Universität Heidelberg, \\ Philosophenweg 12, \\ 69120 Heidelberg, Germany \\ E-mail: Christoph.Werner@physi.uni-heidelberg.de
}

\begin{abstract}
Differential 3-jet cross sections in deep inelastic scattering at low $x$ and $Q^{2}$ have been measured with the $\mathrm{H} 1$ detector at HERA. Three phase-space regions have been selected in order to study parton dynamics from the most global to more restrictive regions of forward jets. The 3 -jet cross sections are compared to fixed order perturbative QCD predictions of $\mathcal{O}\left(\alpha_{\mathrm{s}}^{2}\right)$ and $\mathcal{O}\left(\alpha_{\mathrm{s}}^{3}\right)$ and with two leading order MC predictions. All details of this analysis are described in [1].
\end{abstract}

\section{Introduction}

The $e$-p-collider HERA has opened up a new kinematic regime where the approximations of the DGLAP evolution equations - neglecting terms $\propto \alpha_{\mathrm{s}} \cdot \ln (1 / x)$ which are naturally expected to become large at small $x$ - may no longer be valid. This may lead to a significant enhancement of gluon radiation unordered in transverse momentum compared to DGLAP, expected to be largest for high $P_{\perp}$ forward jets (near to the proton direction). Various measurements [2]- [3] have shown, that the rate of forward jets is indeed higher than predicted by LO QCD predictions including initial state radiation.

This paper concentrates on 3-jet events which require at least one radiated gluon in addition to the two partons from the hard subprocess $\left(\gamma^{\star} g \rightarrow q \bar{q}\right)$ and the comparison with fixed order QCD predictions. The data was also compared to two LO Monte Carlo generators which were able to describe forward jet and dijet production at low $x$.

\section{Experimental Procedure}

3 -jet events in deep inelastic scattering (DIS) have been selected using the inclusive $k_{\perp}$ cluster algorithm in the $\gamma^{\star}-p$ rest frame using data collected 
in 1999 and 2000 with an integrated luminosity of $44.2 \mathrm{pb}^{-1}$. The cross sections are given at the parton resp. stable hadron level. ${ }^{\text {a }}$ They correspond to the following kinematic range: $0.1<y<0.7,4 \mathrm{GeV}^{2}<Q^{2}<$ $80 \mathrm{GeV}^{2}, 10^{-4}<x<10^{-2}$, at least 3 Jets with $E_{\perp \text {, jet }}^{*}>4 \mathrm{GeV}$ and $-1<\eta_{\text {jet }}^{\text {lab }}<2.5$, with the transverse energy sum of the two leading $E_{\perp}$ jets $E_{\perp, \text { jet } 1}^{*}+E_{\perp \text {, jet } 2}^{*}>9 \mathrm{GeV}$ and one jet within the range $-1<\eta_{\text {jet }}^{\text {lab }}<1.3$ in pseudorapidity.

Two LO Monte Carlo programs have been used to compare the data to: RAPGAP which includes standard $k_{\perp}$ ordered parton showers and a resolved photon component and DJANGOH which creates gluon radiation not ordered in $k_{\perp}$ using the color dipole model (CDM).

Fixed order QCD predictions at parton level are calculated using the nlojet++ program which is able to predict 3-jet parton cross sections in leading $\left(\mathrm{LO}, \mathcal{O}\left(\alpha_{\mathrm{s}}^{2}\right)\right)$ and next to leading $\left(\mathrm{NLO}, \mathcal{O}\left(\alpha_{\mathrm{s}}^{3}\right)\right)$ order.

\section{Results}

In Figure 1, the differential cross sections in the number of jets $\left(N_{\text {jet }}\right)$, $x$ and the pseudorapidity of the leading $p_{\perp}^{\star}$ jet are presented for events with 3 or more jets and compared to fixed order QCD predictions in LO and NLO. The jet multiplicity distribution extends up to $N_{\text {jet }}=7$. For this distribution also the predictions of the two LO Monte Carlo programs are shown. It can be noted that the color dipole model (DJANGOH) gives an excellent description of this distribution while RAPGAP fails. The NLO prediction agrees for $N_{\text {jet }}=3$, misses a fraction of 4 -jet events and gives no events with more than 4 jets. This results in a total deficit of $18 \%$.

The other kinematic distributions are not described by the LO QCD prediction neither in shape nor in magnitude. Main discrepancies are seen at low $x$ and for forward jets (large $\eta$ ) where by far too few events are predicted. The NLO prediction improves the situation dramatically in all regions where deviations are observed. The conclusion is therefore that events with more than 3 jets are missing mainly at low $x$ and large $\eta$.

\subsection{Forward Jet Selections}

Here we look at a restricted sample of events with forward jets where the observed differences for the global selection were largest and where the

${ }^{a}$ If not stated otherwise in the plot, the cross sections are given at the level of stable hadrons 
largest sensitivity to unordered gluon radiation is expected. A forward jet is defined in agreement with earlier publications [2] by $\theta_{\text {jet }}<20^{\circ}$ and $x_{\text {jet }}=E_{\text {jet }}^{*} / E_{\mathrm{p}}$, beam $>0.035$. The forward jet sample is further divided into two subsamples: Sample 1 requires two central jets $\left(-1<\eta_{\text {jet }}<1\right)$ and one forward jet, sample 2 requires one central jet, one forward jets and one additional jet with $\eta>1$. Results are shown in figure 2 for the variables $x$ and $\eta_{1}$. The fixed order NLO prediction gives a rather good description for the sample with two central jets, where the step from LO to NLO improves the agreement at low $x$ and large $\eta$ dramatically by more than a factor of 2 , missing only about $30 \%$ of events. The sample with two forward jets on the other hand gives an even more dramatic change reducing the discrepancy at small $x$ from a factor of 10 to 3.5 when going from the LO to the NLO prediction, but a large discrepancy remains.

\section{Comparison to the LO Monte Carlo Programs}

Finally we compare to the two LO Monte Carlo Programs RAPGAP and DJANGOH to the measured cross sections at hadron level. Figure 2 (right) shows the comparison for the 3-jet cross sections in the Bjørken scaling variable $x$ (top) and the difference of the pseudorapidity of the two leading $P_{\perp}^{*}$ jets $\left(\eta_{1}-\eta_{2} \mathrm{i}\right.$, bottom) after normalising both $\mathrm{MC}$ sets to the data. RAPGAP fails to describe the data, while DJANGOH compares remarkably well.
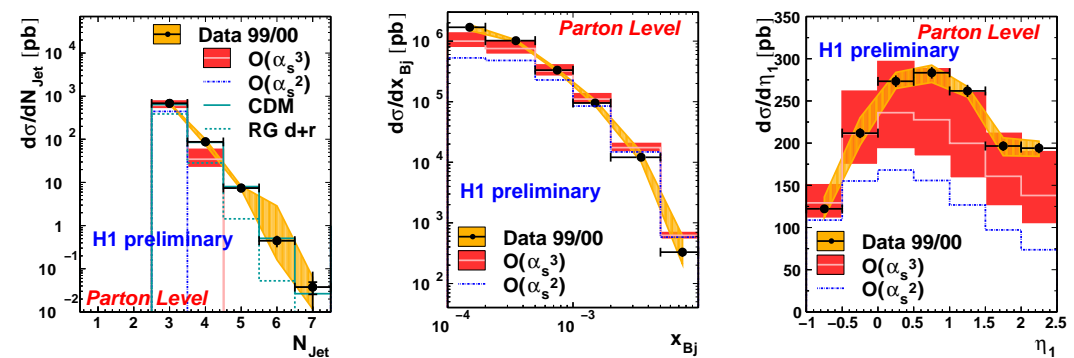

Figure 1. Differential cross sections in the number of jets $N_{\text {jet }}$ (left), the Bjørken scaling variable $x$ (mid) and the pseudorapidity of the leading $p_{\perp}^{\star}$ jet $\eta_{1}$ (right). The inner error bars represent the statistical error of the data, the total error bars correspond to the statistical and uncorrelated sytematic errors added in quadrature. The (orange) hatched error bands show the estimate of the correlated systematic uncertainties. The data has an additional overall normalisation error of $19 \%$ (not displayed). The shaded (red) band shows the NLO prediction where the size of the band indicates the scale uncertainty of the NLO calculation, the dashed dotted line represents the LO prediction. The data for $N_{\text {jet }}$ are also compared to the two LO Monte Carlo programs RAPGAP (dotted line) and DJANGOH (CDM) (solid line). 


\section{Summary}

A LO MC with addition of non $k_{\perp}$-ordered gluon radiation as implemented in the color dipole model (CDM) gives a remarkably good description of the 3 -jet events and even of higher multiplicities. The most remarkable result of the present analysis however is the success of the fixed order QCD prediction in $\mathcal{O}\left(\alpha_{\mathrm{s}}^{3}\right)$ closing most of the gap between the data and the $\mathcal{O}\left(\alpha_{s}^{2}\right)$ prediction. Remaining discrepancies are concentrated at $x$ values below $10^{-3}$ and events where two jets are going forward. This is a topology which is expected to be most sensitive to gluon radiation We conclude therefore that unordered gluon emission plays a significant role at low $x$.

\section{References}

1. http://www-h1.desy.de/h1/www/publications/htmlsplit/H1prelim-06-034.long.html

2. A. Aktas et al. [H1 Collaboration], arXiv:hep-ex/0508055.

3. S. Chekanov et al. [ZEUS Collaboration], arXiv:hep-ex/0502029.
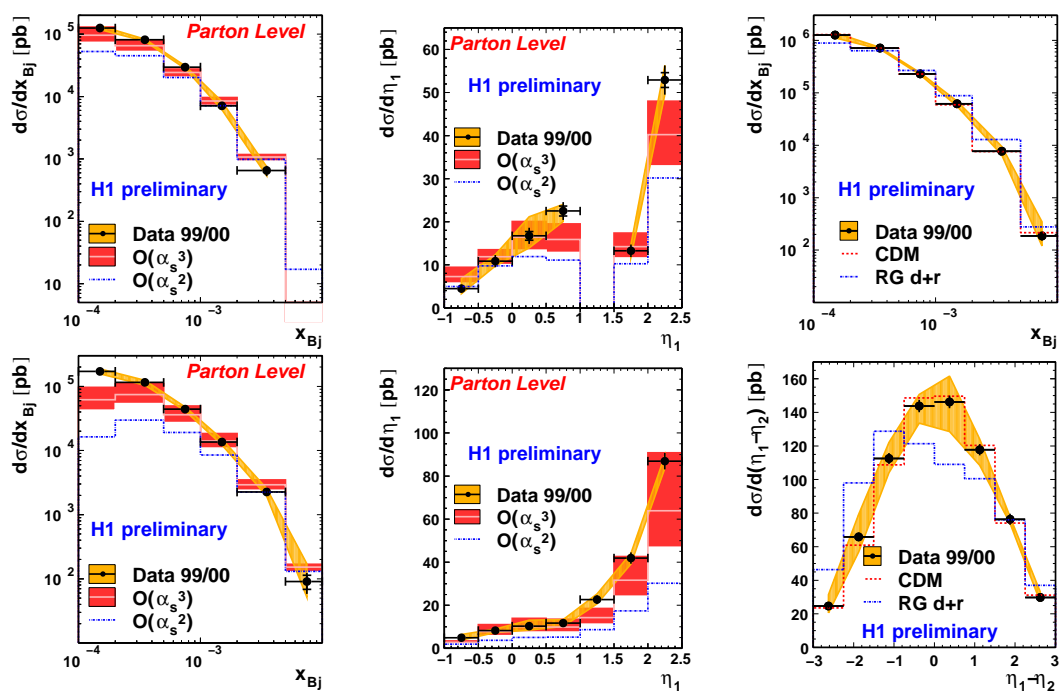

Figure 2. Differential cross sections in $x$ (left) and pseudorapidity (mid) of the leading jet for the samples with two central (top) resp. two forward jets (bottom). Other details are as in the caption to fig. 1 The two plots on the right are differential cross sections at hadron jet level in the Bjørken scaling variable $x$ and the difference of the pseudorapidities of the two leading jets. The inner error bars represent the statistical error of the data, the total error bars correspond to the statistical and uncorrelated systematic errors added in quadrature. The correlated systematic errors are shown by the hatched error band. The data are compared to the two LO Monte Carlo programs DJANGO (CDM) (dashed line) and RAPGAP (dashed dotted line). Both Monte Carlo cross sections are scaled to the data cross sections by factors of 1.08 (DJANGOH) resp. 1.74 (RAPGAP) 